\title{
Aeroelastic Response and Protection of Space Shuttle External Tank Cable Trays
}

\author{
John W. Edwards, ${ }^{*}$ Donald F. Keller, ${ }^{\dagger}$ David M. Schuster, ${ }^{\ddagger}$ David J. Piatak, ${ }^{\S}$ Russ D. Rausch, ${ }^{* *}$ \\ Robert E Bartels, ${ }^{\dagger \dagger}$ Thomas G. Ivanco, ${ }^{\sharp}$ Stanley R. Cole, ${ }^{\S}$ and Charles V. Spain ${ }^{* * *}$ \\ Aeroelasticity Branch, NASA Langley Research Center, Hampton, Virginia, 23681
}

Sections of the Space Shuttle External Tank Liquid Oxygen $\left(\mathrm{LO}_{2}\right)$ and Liquid Hydrogen $\left(\mathrm{LH}_{2}\right)$ cable trays are shielded from potentially damaging airloads with foam Protuberance Aerodynamic Load (PAL) Ramps. Flight standard design $\mathrm{LO}_{2}$ and $\mathrm{LH}_{2}$ cable tray sections were tested with and without PAL Ramp models in the United States Air Force Arnold Engineering Development Center's (AEDC) 16T transonic wind tunnel to obtain experimental data on the aeroelastic stability and response characteristics of the trays and as part of the larger effort to determine whether the PAL ramps can be safely modified or removed. Computational Fluid Dynamic simulations of the full-stack shuttle launch configuration were used to investigate the flow characeristics around and under the cable trays without the protective PAL ramps and to define maximum crossflow Mach numbers and dynamic pressures experienced during launch. These crossflow conditions were used to establish wind tunnel test conditions which also included conservative margins. For all of the conditions and configurations tested, no aeroelastic instabilities or unacceptable dynamic response levels were encountered and no visible structural damage was experienced by any of the tested cable tray sections. Based upon this aeroelastic characterization test, three potentially acceptable alternatives are available for the $\mathrm{LO}_{2}$ cable tray PAL Ramps: MiniRamps, Tray Fences, or No Ramps. All configurations were tested to maximum conditions, except the $\mathrm{LH}_{2}$ trays at $\mathbf{- 1 5}$ deg. crossflow angle. This exception is the only caveat preventing the proposal of acceptable alternative configurations for the $\mathbf{L H}_{2}$ trays as well. Structural assessment of all tray loads and tray response measurements from launches following the Shuttle Return To Flight with the existing PAL Ramps will determine the acceptability of these PAL Ramp alternatives.

\section{Nomenclature}

\footnotetext{
${ }^{\prime} c_{p}{ }^{\prime}=$ normalized pressure coefficient

' $g$ ' $\quad=$ normalized acceleration

$q \quad=$ dynamic pressure, pounds per square foot
}

\footnotetext{
${ }^{*}$ Senior Research Engineer, Fellow, AIAA.

${ }^{\dagger}$ Senior Research Engineer.

* Senior Research Engineer, Associate Fellow AIAA.

${ }^{\S}$ Senior Research Engineer.

** Senior Research Engineer, Member, AIAA.

"† Senior Research Engineer, Member, ASME.

Aerospace Engineer, Member, AIAA.

$\S \S$ Branch Head, Associate Fellow, AIAA.

${ }^{* * *}$ Senior Research Engineer.
}

This material is declared a work of the U. S. Government and is not subject to copyright protection in the United States. 


\section{Introduction}

\section{A. Problem Background}

The recent STS-107 Shuttle accident has raised concerns about the integrity of the foam Protuberance Air Load (PAL) Ramps that protect the External Tank (ET) liquid oxygen $\left(\mathrm{LO}_{2}\right)$ tank, and liquid hydrogen $\left(\mathrm{LH}_{2}\right)$ tank cable trays from high velocity cross-flows. The External Tank PAL Ramp Evaluation Project was undertaken to determine if the PAL ramps can be removed or modified to minimize the quantity of foam in these areas and thus reduce the risk of debris to the Shuttle Orbiter.

A study in $1981^{1}$ showed the potential of aeroelastic instability of the original unprotected cable trays. As a result of this study, foam PAL ramps were used on the first and all subsequent flight tanks to shield the cable trays from the perceived damaging airloads (see Figures 1 and 2). Further studies in $1982^{2}$ and $1983^{3}$ investigated several fixes to replace the current PAL ramps while mitigating possible cable tray aeroelastic instability problems. Though identifying promising alternate fixes, the results of these studies were not sufficient to replace the existing PAL ramps. Both the $\mathrm{LO}_{2}$ and $\mathrm{LH}_{2}$ cable tray designs were modified for the ET Light Weight Tank (LWT) development and remained unchanged for the Super Light Weight Tank (SLWT). The original PAL ramps continued to be employed in these redesigns and thus reduce the risk of foam debris for the Shuttle Orbiter.

In the summer of 2003 the External Tank PAL Ramp Project Team was formed to investigate the aeroelastic stability and response of the $\mathrm{LO}_{2}$ and $\mathrm{LH}_{2}$ cable trays shielded by the existing PAL ramps and evaluate modification or elimination of the foam PAL ramps. The plan prepared by the team focused upon two approaches: 1.) a reanalysis of the current cable tray stability utilizing the original aeroelastic analysis, which had led to the installation of the ramps and 2.) a wind tunnel test of flight standard design cable tray sections to establish their aeroelastic stability and structural response characteristics under launch conditions.

\section{B. External Tank PAL Ramps Evaluation Project}

The original aeroelastic analysis utilized the then state-of-the-art analysis capability in the launch vehicle community: two-dimensional, component, stability analyses using conservative, quasi-steady, assumed, aerodynamic loading functions. Dynamic wind tunnel component testing was to have provided necessary quasisteady stability derivative information for the planned reanalysis. However, a model/tunnel interference issue was encountered which terminated this cable tray stability reanalysis.

Computational capability for this type of analysis has advanced greatly in the intervening years. Still, the complexity of the aerodynamic flow about the cable trays on the Space Shuttle launch configuration precluded a direct Computational Aeroelastic (CA) analysis approach utilizing the most advanced Computational Fluid Dynamic

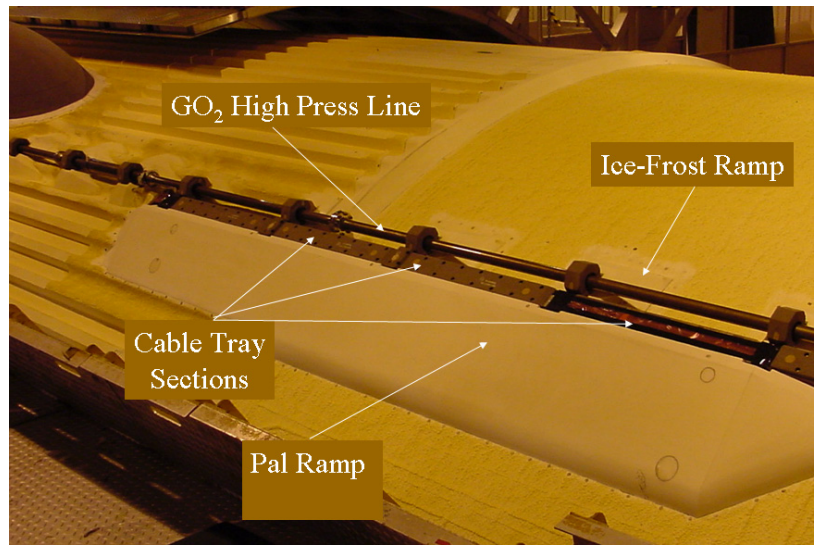

Figure 1. Liquid Oxygen cable trays, Gaseous Oxygen pressurization line, and $\mathrm{LO}_{2}$ PAL ramp installed on external tank. The three cable tray sections tested at AEDC 16T (from ET station 760.2 to 862.0) are indicated.

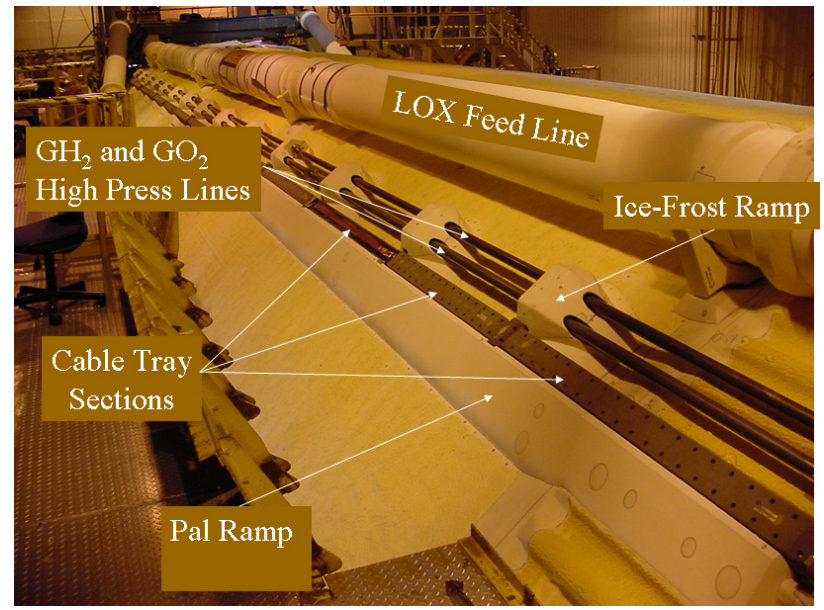

Figure 2. Liquid Hydrogen cable trays, Gaseous Oxygen and Hydrogen pressurization lines, and $\mathrm{LH}_{2}$ PAL ramp installed on external tank. The three cable tray sections tested at AEDC 16T (from ET station 1082.0 to 1270.2) are indicated. 
(CFD) codes. Such computations have become commonplace for steady flow conditions but not for the highly unsteady, separated flows which would be required for accurate aeroelastic stability and response calculations. Also, there is a very large gap between the demonstration of individual CA analysis cases and a proven design capability.

Accordingly, the team focused upon a second direct approach to evaluating aeroelastic response of the cable trays and proposed PAL ramp modifications: aeroelastic wind tunnel response tests of sections of the flight standard cable trays at the highest loading conditions encountered during launch (including suitable margins). Three sections each of the $\mathrm{LO}_{2}$ and $\mathrm{LH}_{2}$ cable trays were tested in the $16 \mathrm{~T}$ transonic wind tunnel at the U. S. Air Force Arnold Engineering Development Center (AEDC) in Tullahoma, Tennessee during the period of August to November 2004 (AEDC Test TF1003). Figures 3 and 4 show the $\mathrm{LO}_{2}$ cable tray and $\mathrm{LH}_{2}$ cable tray Test Articles installed in the wind tunnel test section.

The tray section Test Articles were installed with models of the ice-frost ramp mounting supports and included flight standard Gaseous Oxygen

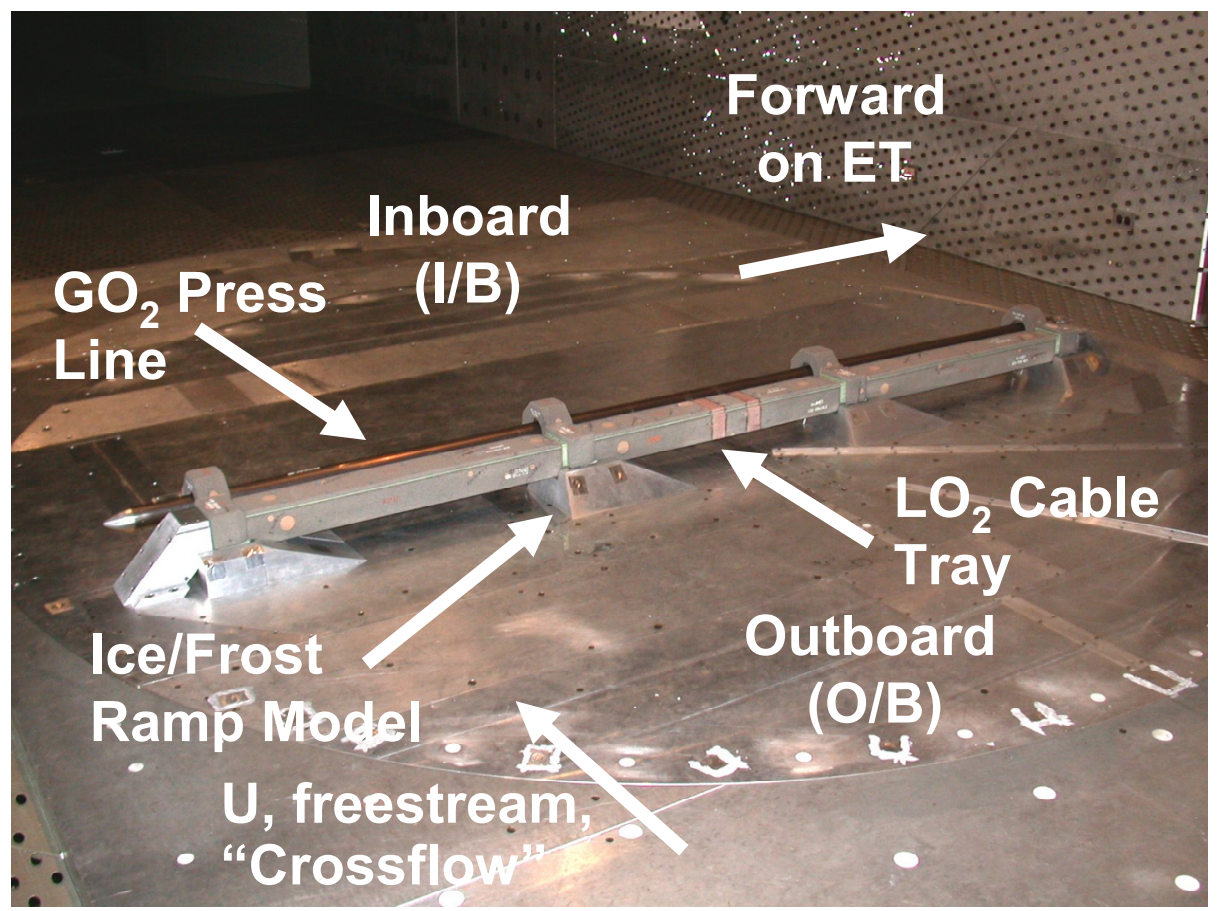

Figure 3. $\mathrm{LO}_{2}$ cable tray test article without ramps or fence, installed in the test section at $90^{\circ}$ crossflow angle.

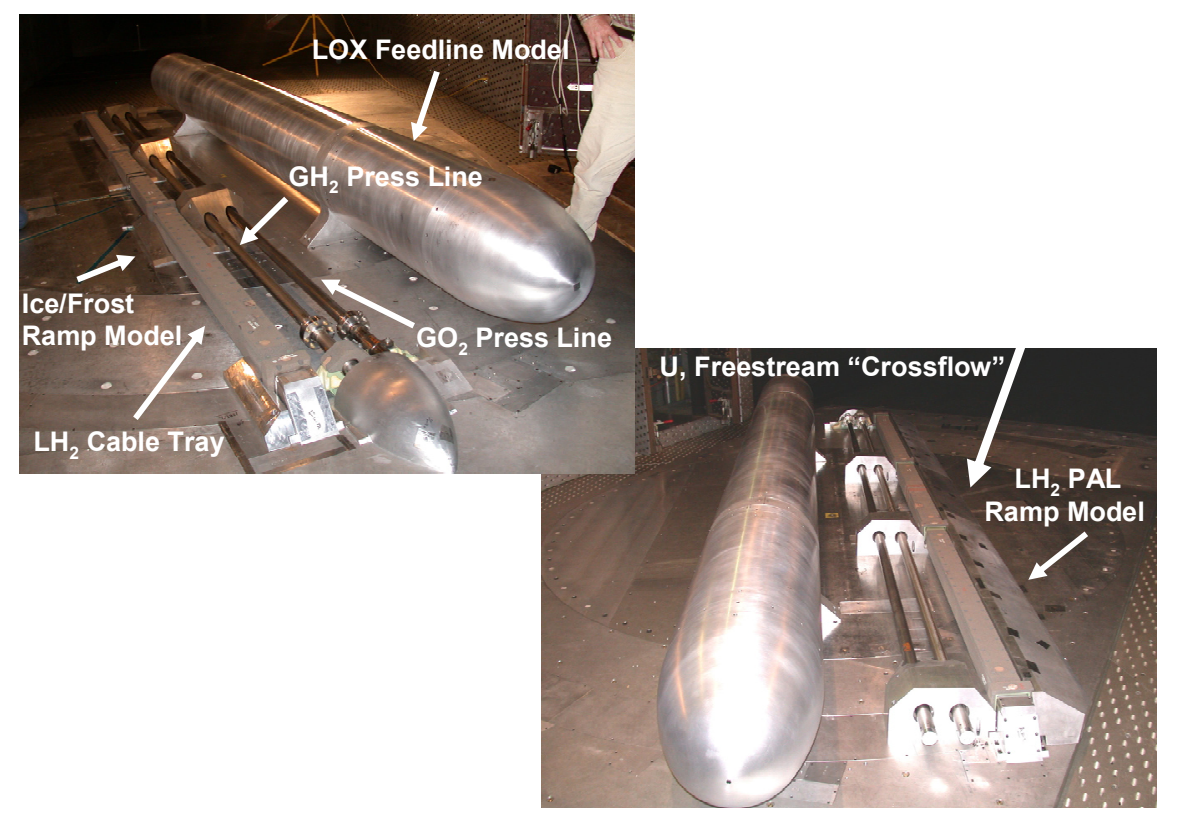

Figure 4. $\mathrm{LH}_{2}$ cable tray test article with no ramps and with mini-ramps, installed in the tunnel at $30^{\circ}$ crossflow angle.

$\left(\mathrm{GO}_{2}\right)$ and Hydrogen $\left(\mathrm{GH}_{2}\right)$ pressure supply pipes. The $\mathrm{LH}_{2}$ tray configurations included a 17 inch diameter aluminum model of the ET Liquid Oxygen (LOX) supply line. The trays and supporting structures were mounted on an 11.5 foot diameter aluminum floor plate which was itself mounted in a $12 \times 20 \times 0.25$ foot plate structure on the floor of the 16T tunnel test section. The floor plate could be rotated to produce the desired crossflow angles of the tunnel flow over the trays. Both $\mathrm{LO}_{2}$ and $\mathrm{LH}_{2}$ trays were tested with the existing PAL Ramps (PR), with smaller PAL ramps (Mini-Ramps, MR) and with No Ramps (NR). A third $\mathrm{LO}_{2}$ tray Test Article with a trailing-edge fence extending beneath the tray was also tested. The $\mathrm{LO}_{2}$ tray Test Articles were tested at crossflow angles of 90, 60, and 30 degrees., while the $\mathrm{LH}_{2}$ tray Test Article was tested at $+30,+15$, and -15 degrees. Finally, the $\mathrm{LO}_{2}$ trays were 
tested at varying tray rotation, or pitch, angles. The entire test consisted of 26 of these tray/ramp/crossflow angle/pitch configurations.

While the AEDC 16T tests did involve the flight standard cable trays, the effects of the many components of the launch configuration (External Tank and its flexibility, Solid Rocket Boosters, Shuttle, etc) were not included. Thus the test was an aeroelastic characterization test of the cable trays rather than an aeroelastic stability clearance test. The combination of these test results, flight data of cable tray structural response from upcoming launches, and tray response to existing ET base loads will be used to evaluate the suitability of proposed PAL ramp modifications or elimination (Steps 6 and 7 of Project Outline, shown in Figures 5 and 6).

All of the flight standard design cable tray sections survived all planned test conditions and encountered no instabilities, no structural damage, and no unacceptable structural responses. All test configurations of the $\mathrm{LO}_{2}$ cable trays achieved the desired maximum Mach numbers and dynamic pressures (which included margins of 45 percent over maximum predicted launch conditions). All but three of the $\mathrm{LH}_{2}$ cable tray test configurations (at -15 degrees crossflow angle) also achieved these goals. The desired maximum dynamic pressures for these three $\mathrm{LH}_{2}$ cable tray test configurations were outside the operational capabilities of the wind tunnel. Still, these three $\mathrm{LH}_{2}$ configurations did achieve 70 percent of the desired conditions (encompassing the calculated maximum launch conditions) with no indication of stability or response issues.

Figures 5 and 6 give the project outline which was developed to guide decisions for the modification or removal of the PAL Ramps. Seven steps were identified for the several interlocking portions of the project which provide the information needed for a decision to implement PAL Ramp modifications or removal:

1.) Ground Vibration Test (GVT) Surveys of the cable trays were performed (Section IV of this paper). Finite Element Models of the cable trays and their mounting systems were developed.

2.) Several subscale, component wind tunnel tests were planned to determine steady airloads and stability derivatives on the cable trays. Potential tray section modifications to alleviate concerns of adverse aeroelastic behavior were investigated, leading to selection of a candidate trailing-edge fence design. Tests to measure dynamic stability derivative information were also attempted (Section III-C).

3.) Steady Computational Fluid Dynamics code simulations of models of the full stack shuttle launch configuration were data-mined to determine cable tray maximum inflow conditions (Section V).

4.) A full scale wind tunnel test of flight design standard cable trays at maximum airload conditions experienced during launch was conducted. The test was to establish the aeroelastic stability behavior of the trays and the aeroelastic response characteristics of the trays (including suitable margins, Section VI). Tray acceleration test termination criteria were established to provide for wind tunnel safety.

5.) Instrumentation of launch configuration cable trays is planned to establish behavior of trays with the existing PAL Ramps.

6.) Structural Assessment of the cable trays with the current PAL Ramps, Ramp modifications, and Ramps removed will include all information normally utilized for structural clearance of the trays (steady airloads, existing base-drive response, dynamic characteristics). Additional information will include the unsteady airload response measured in the wind tunnel test and the current configuration flight response.

7.) Aeroelastic assessment of the cable trays in conjunction with the several Ramp configurations was the responsibility of the NASA Langley Research Center (LaRC) Aeroelasticity Branch. The assessment involves two main components: 1.) the aeroelastic stability of the trays including marginal stability conditions, and 2.) (assuming the stability of the trays) the aeroelastic response levels of the trays (Sections VI-F, and G). 


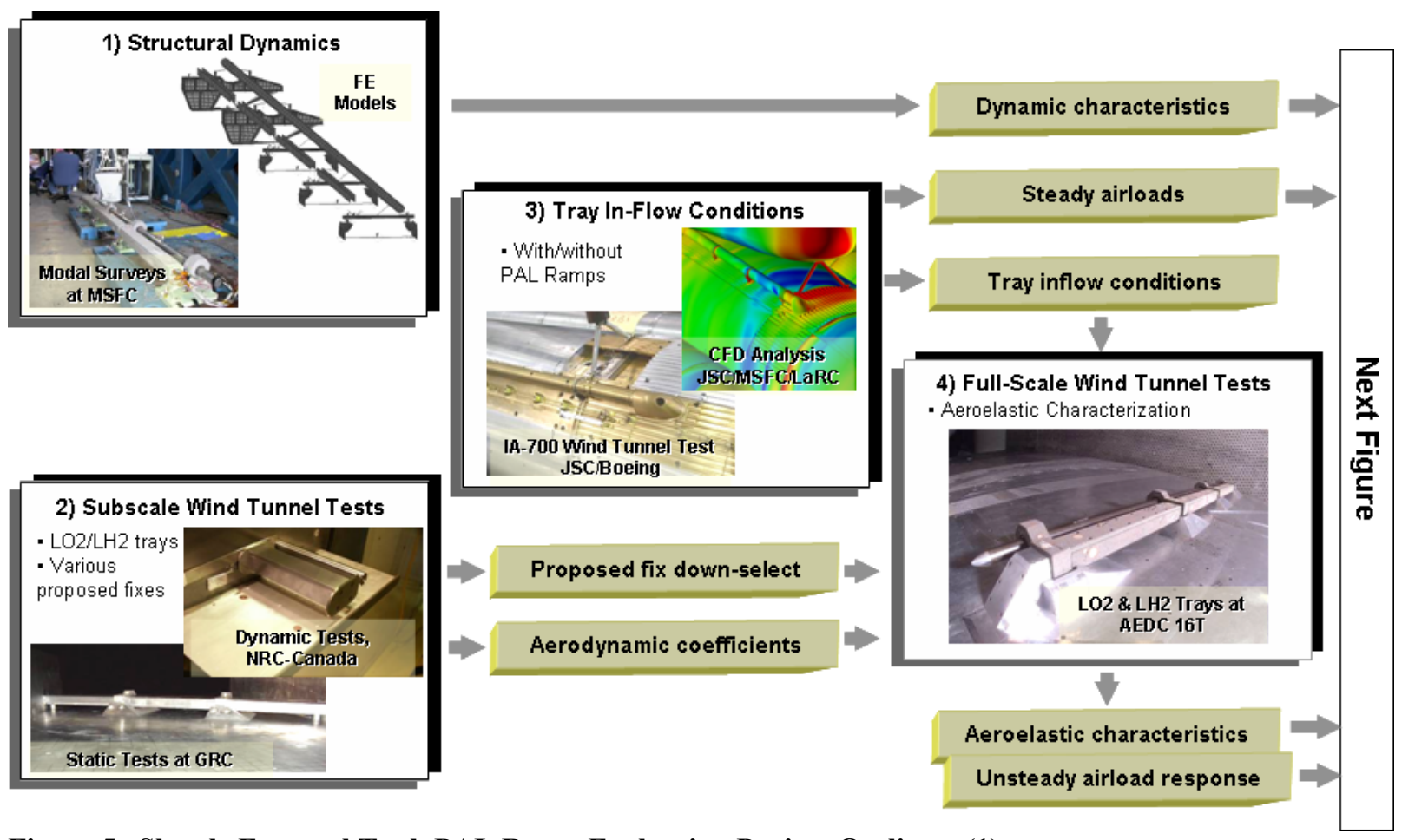

Figure 5. Shuttle External Tank PAL Ramp Evaluation Project Outline - (1).

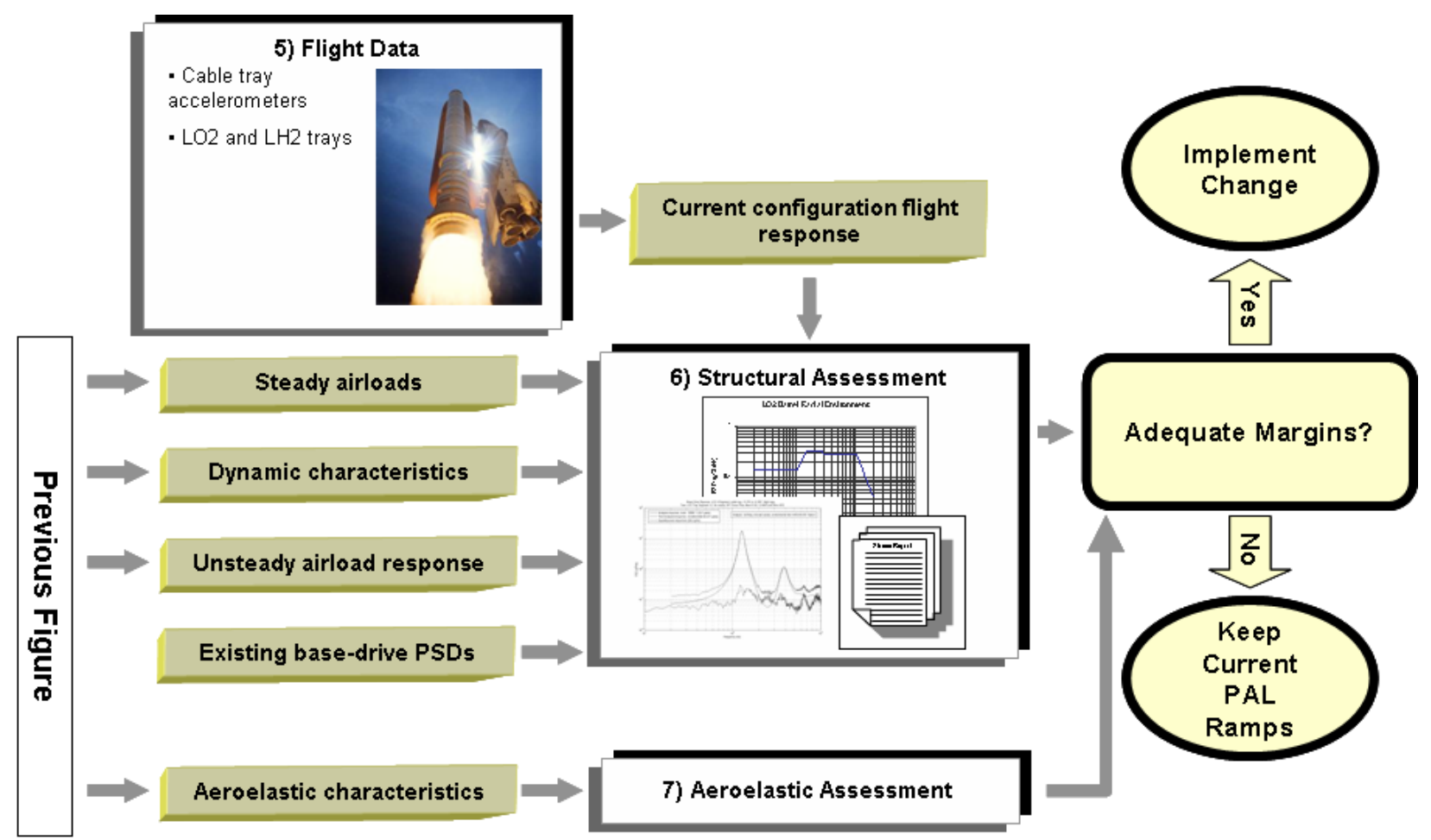

Figure 6. Shuttle External Tank PAL Ramp Evaluation Project Outline - (2). 


\section{Original Stability Analysis of the $\mathrm{LO}_{2}$ and $\mathrm{LH}_{2}$ Cable Trays}

The concern over the aeroelastic stability of the cable trays arose from interpretation of oil flow photographs from a $0.4 \%$ scale model of the full stack shuttle launch configuration tested in the NASA Marshall Space Flight Center 14inch Transonic Wind Tunnel (TWT) in the late 1970's (Figure 7). The bow shock wave of the Solid Rocket Booster impinges upon the $\mathrm{LO}_{2}$ cable trays, turning the flow behind the shock and generating a region of crossflow over the trays. The shock and this crossflow region move aft with increasing Mach number.

Then state-of-the-art aeroelastic analysis and design capability for such bluff-body structures as the cable trays was limited. In the launch vehicle community this consisted of conservative, empirical, quasi-steady analysis of two-dimensional 'typical sections'. Static stability derivatives were measured in wind tunnel tests on simplified 2-D models of cable tray cross sections. Later, dynamic stability derivatives were measured by Canada's NAE Laboratory for the original Heavy Weight Tank (HWT) $\mathrm{LO}_{2}$ tray section which had a different cross section than the current cable tray. The crosssections of this original tray and the current SLWT tray are compared in Figure 8. From discontinuous loading seen in

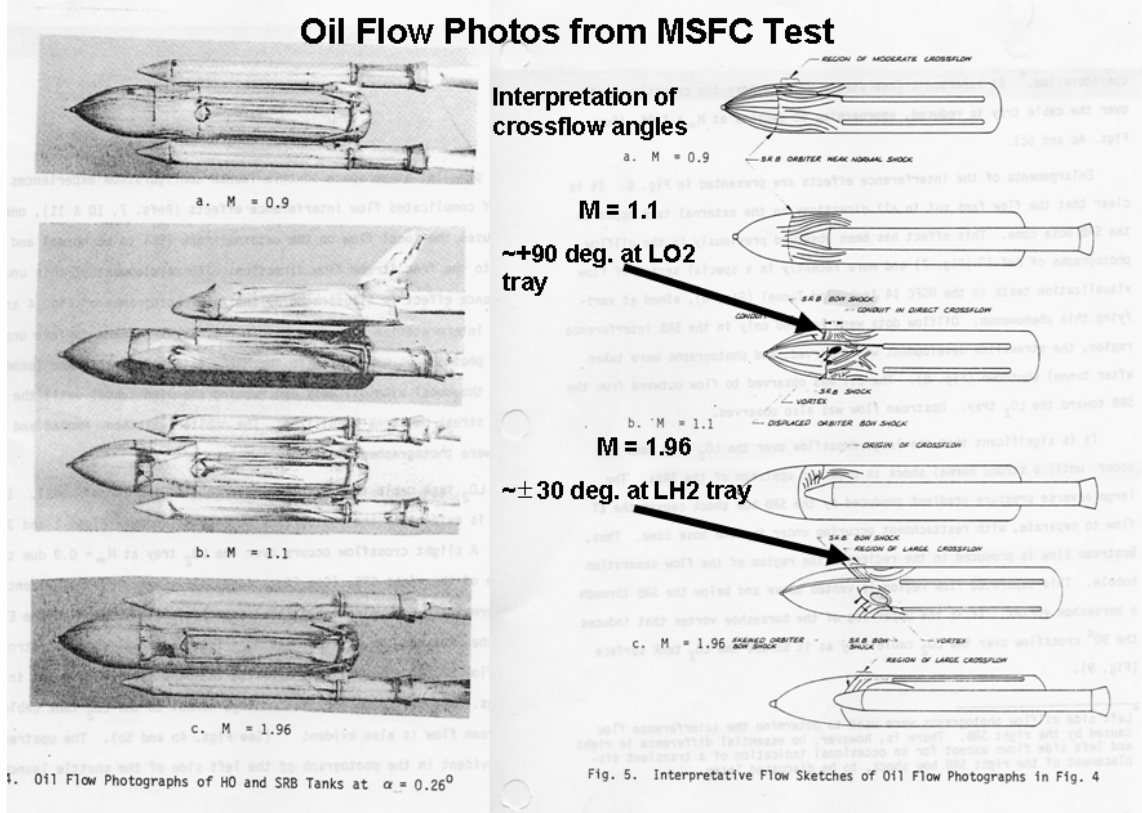

Figure 7. $0.4 \%$ scale model of space shuttle full-stack launch configuration tested in NASA MSFC's 14 inch wind tunnel and interpretative sketches of oil flow patterns.

the static stability derivatives and in tray pressures, several nonlinear loading mechanisms were inferred:

- sudden flow separation and reattachment in the gap between the tray and the tunnel floor (external tank surface) as tray angle was varied at transonic and supersonic speeds (simulating transonic and supersonic crossflow velocities for the full launch configuration);

- discontinuous loading on top of the tray as supersonic flow separation bubbles form, lengthen, and collapse;

- the possibility of vortex burst loading on the top face of the cable trays was considered in the stability analysis of the $\mathrm{LH}_{2}$ trays.

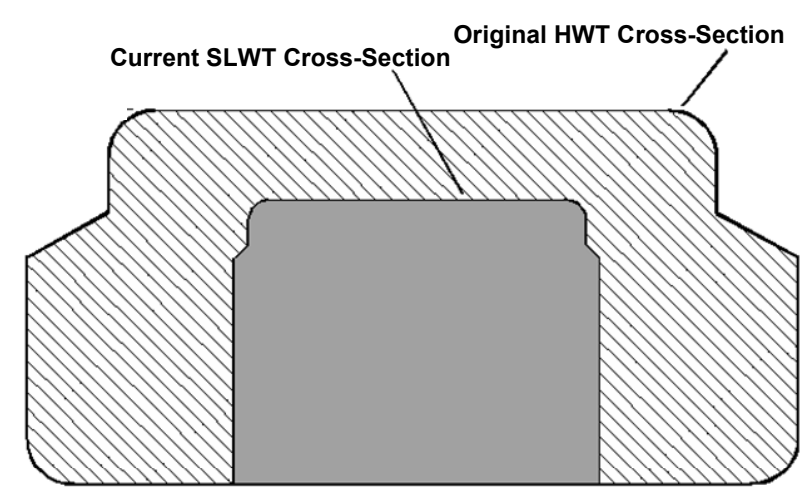

It is important to understand the limited predictive capability both then and now for unsteady airloads on such bluff bodies in high-speed crossflows. There was very limited experimental data of unsteady pressures or loads on bodies resembling the cable tray cross-sections in high-speed flows, particularly in the context of a gap or channel beneath the body (the trays are supported by mounts 2-2.5 inches off of the external tank surface and the mounts are shielded by complex faceted, foam ice/frost ramps). Available data, mostly from 2-D solid models of geometries like rectangular prisms, were generally for low-speed flows. Available data from cylindrical, launch vehicle geometries were also used for reference. Compressibility corrections from streamlined airfoil databases were utilized. A series 
of transonic wind tunnel tests were made on 2-D models of the original $\mathrm{LO}_{2}$ tray cross-section and the succeeding LWT $\mathrm{LH}_{2}$ tray. Steady force, moment, and pressure measurements were obtained.

In 1981 dynamic stability derivative tests of the HWT $\mathrm{LO}_{2}$ tray were conducted at the NRC Unsteady Aerodynamics Laboratory (Reference 2). The assumed tray modes of motion, plunging and pitching, were studied separately. Discontinuous static lift and moment coefficients (versus angle) were modeled. Unsteady phase lags due to modal velocities which are critical to instability mechanisms were modeled empirically. These analyses led to predictions of tray instabilities and limit cycle oscillations with unacceptably large amplitudes. Wind tunnel tests then studied methods of shielding the trays from the crossflow in order to alleviate or eliminate the nonlinear loading mechanisms which produced the instabilities, resulting finally in the selection of the current PAL ramp configurations. Details of these early wind tunnel tests and the studies undertaken concerning the aeroelastic stability of the trays are reported in Reference 3 for the $\mathrm{LO}_{2}$ cable trays and for the $\mathrm{LH}_{2}$ cable trays.

This brief outline of the original aeroelastic stability analysis procedures is meant to emphasize the conservative assumptions which were necessary in the face of the difficult design conditions and the limited knowledge of the unsteady loads on the cable trays in order to provide an acceptable level of safety for the External Tank and the Space Shuttle. It is important to note that there were no tests of flexible or aeroelastic 3-D cable tray models prior to this test of flight standard cable trays in the AEDC 16T transonic wind tunnel discussed herein.

\section{Steady and Unsteady 2-D Component CFD Computations}

Two-Dimensional (2-D) CFD calculations of cable tray cross sections have been utilized to study steady and unsteady airloads. Unsteady CFD component calculations have also been applied to the well-known phenomenon of self-induced vortex shedding about bluff bodies. These 2-D computations idealize the situation of $90 \mathrm{deg}$. crossflow over the mid-span regions of the cable tray sections, whereas the 3-D influence of the ice-frost ramps supporting the section ends leads to highly 3 -D flows. At these conditions Strouhal vortex shedding occurs at $\sim 500-600 \mathrm{~Hz}$. for high subsonic-transonic crossflows. This frequency range is well above the lowest frequencies of the cable trays (see Section IV) and the coupling of Strouhal vortex shedding and cable tray structural motions was not anticipated nor observed in the wind tunnel test.

A more complicated situation arises for swept crossflows over the cable trays where there would be the potential of lower velocity normal (90 deg.) velocity components across the cable trays and inducing lower frequency vortex shedding. Such behavior is also much more difficult to simulate with CFD calculations. The flow is inherently 3-D and unsteady and no such CFD calculations have been made. Such Strouhal shedding resonance features were observed in the wind tunnel test and are discussed.

A high-priority condition for this problem is the $\mathrm{LO}_{2}$ tray section operating at 90 deg. crossflow angle. At this crossflow condition, the tray sees the full impact of the oncoming local flow without any relief due to the crossflow angle. In addition, the flow is not heavily influenced by upstream cable tray geometry components, such as the ice frost ramps, so it is postulated that a 2-D simulation of the cable tray system might be used to investigate the impact of various flow and geometric parameters on the cable tray loads. NASA engineers formulated a set of CFD computations to capture and simulate unsteady flow effects as well as the effect of the incoming tank (wind tunnel floor) boundary layer on the cable tray loads. The CCFL3D code analysis method used a block structured grid approach to the modeling of the geometry. The grid used in the analysis of the $\mathrm{LO}_{2}$ cable tray and $\mathrm{GO}_{2}$ repressurization line is shown in Figure 9. The ability of the viscous floor boundary layer in the wind tunnel to adequately simulate the viscous boundary layer experienced by the cable trays on the External Tank during launch was also

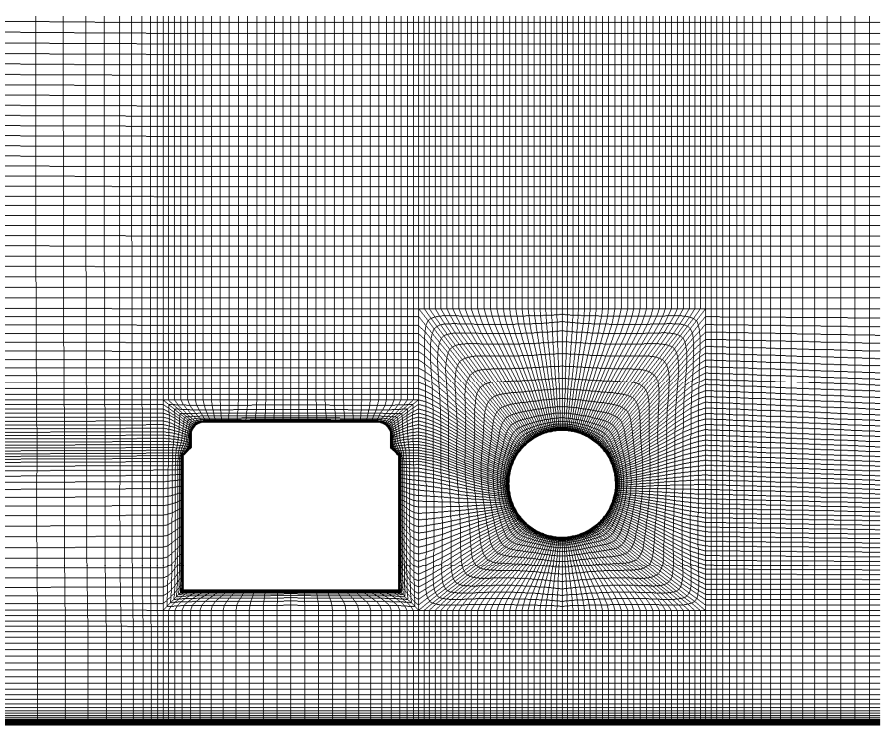

Figure 9. CFL3D block grid system in vicinity of $\mathrm{LO}_{2}$ cable tray. 

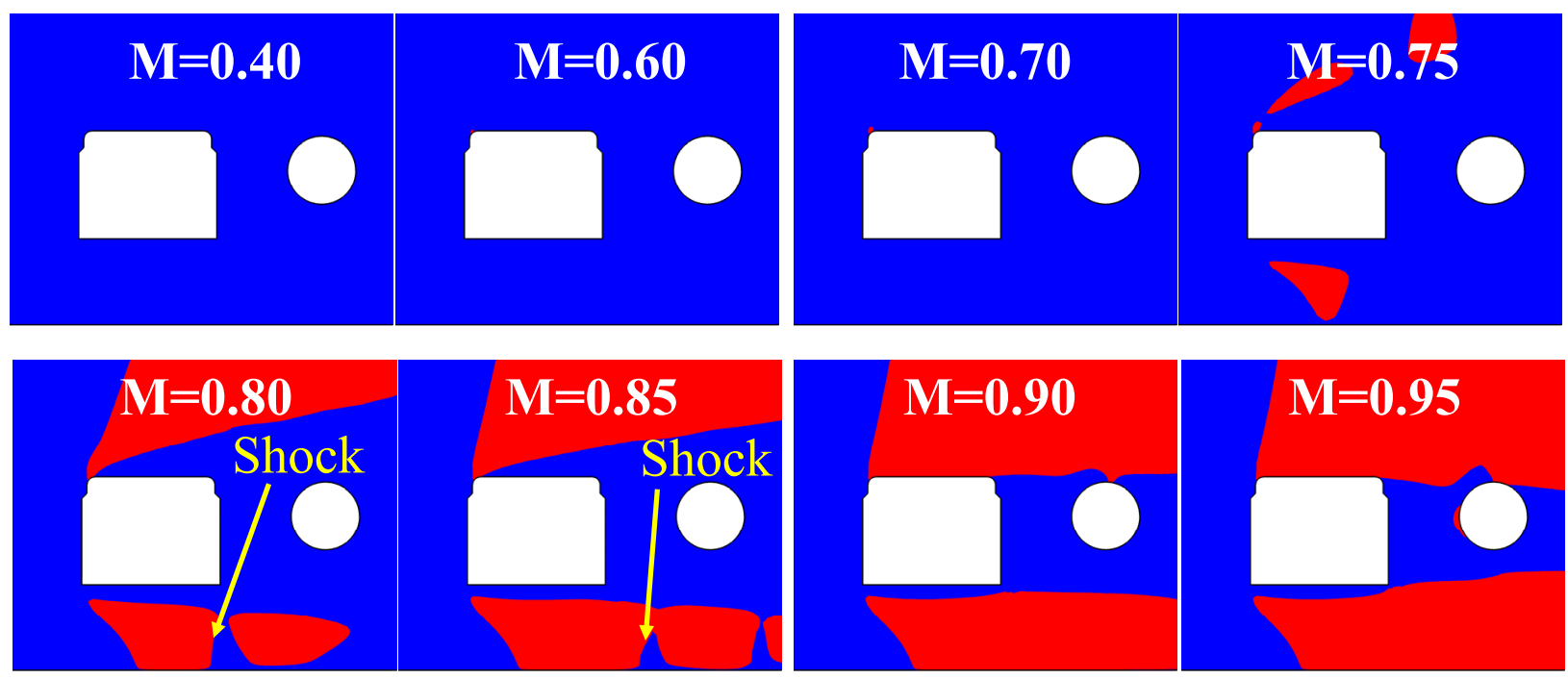

Figure 10. . $\mathrm{LO}_{2}$ tray transonic flow maps as a function of freestream Mach number, viscous tank. (Blue subsonic, Red - supersonic).

investigated with CFD.

Steady transonic flow maps for the viscous tank case are shown in Figure 10. Blue shading signifies subsonic flow, while red shading shows supersonic flow. A weak shock is present in the gap flow at $\mathrm{M}=0.75,0.80$ and 0.85 . At $\mathrm{M}=0.80$, the shock sits just under the aft edge of the tray, while at $\mathrm{M}=0.85$, the shock has moved farther aft to the repressurization line vicinity. The acceleration around the tray leading edge causes separation on the tray lower surface and the flow there remains subsonic all the way to the tray trailing edge.

A series of time-accurate computations were performed to study the anticipated unsteady

Strouhal vortex shedding behavior. The time accurate simulations predict that all cases below $\mathrm{M}=0.85$ produce unsteady, periodic flow around the $\mathrm{LO}_{2}$ cable tray system. Visualization of flowfield animations of the time accurate solutions indicate that this unsteadiness is a Strouhal vortex shedding phenomenon that is eventually quenched at higher Mach numbers by the appearance of large areas of supersonic flow surrounding the cable tray and repressurization line. Figure 11 shows a series of normalized normal-force coefficient time histories from the time accurate computations for Mach numbers between 0.60 and 0.80. At these four Mach numbers, the onset of unsteady flow is very rapid, while for lower Mach numbers, the onset is much more gradual and requires long run times to fully develop. At $\mathrm{M}=0.85$, not shown here, the amplitude of oscillation is significantly reduced and at $\mathrm{M}=$ 0.90 and 0.95 , the time accurate simulations predict steady flow.

Figure 12 shows the variation of the Strouhal vortex shedding frequency on the $\mathrm{LO}_{2}$ tray system as a function of Mach number for the inviscid and viscous tank conditions simulated with CFD and the AEDC 16T experimental test data. The experimental data were acquired during testing of the tray system at AEDC 16T at 90 degree cross flow with no PAL ramp. The agreement between experimental and computed frequencies is excellent.

\section{Component Testing}

There have been a number of wind tunnel tests supporting this effort. Most have been static component tests of $\mathrm{LO}_{2}$ and $\mathrm{LH}_{2}$ scaled model configurations. There have also been full stack shuttle launch configuration static model tests 
and one dynamic scaled model component test.

\section{A. Full Stack Shuttle Launch Configuration Tests}

A $0.4 \%$ full-stack shuttle launch configuration model was tested in the NASA MSFC 14-inch TWT before the first launch of the shuttle. Oil flow photographs from this test were instrumental in the installation of the PAL ramps. Following the STS-107 crash and a review of these photographs, limited testing of this model was again undertaken. At this time large differences of results from this $0.4 \%$ scale model were noted in comparison with results from a 3\% scaled model tested early in the shuttle program and with new CFD computations for the full-stack shuttle launch configuration. There was better agreement between the CFD and 3\% scaled model results than there was between the CFD and the $0.4 \%$ scale model results, highlighting concerns over lack of Reynolds number similitude, laminar or transitional flow issues, and tunnel blockage issues for the small-scale model. This also impacts conclusions concerning crossflow behavior about the cable trays drawn from the original oil flow photographs.

The 3\% scaled model was retested in the fall of 2004. Two sections each of the $\mathrm{LO}_{2}$ and $\mathrm{LH}_{2}$ cable trays were instrumented with balances and were tested with and without PAL ramps. Static load data from these tests is being evaluated by the ET PAL Ramp Evaluation team.

\section{B. Steady Two-Dimensional Component Tests}

The ET PAL Ramp Evaluation Project has performed a number of steady cable tray component wind tunnel tests. The goal was improved understanding of the aerodynamic flow field around the trays and to support the planned stability reanalysis of the cables trays using the original quasi-steady tray stability analysis method. Static aerodynamic stability lift and pitching moment coefficients were obtained from these tests as well as pressure distributions and flow visualizations.

\section{Dynamic Two-Dimensional Component Test}

Measurement of dynamic stability derivatives was required in order to complete the planned stability analysis of the $\mathrm{LO}_{2}$ cable trays using the original quasi-steady stability method. This information was to be measured in the Canadian NRC $5 \mathrm{ft}$. by $5 \mathrm{ft}$. transonic wind tunnel. A $5 \mathrm{ft}$. x 15 in. test section insert was used for this "twodimensional" component model test. The model was mounted on either pitch or plunge spring flexures and driven in oscillatory motion by an electromagnetic vibrator. The six-inch span model and slightly larger ground plane, both with endplates, were mounted from one wall of the 15 inch channel. With the different cross section geometry of the current SLWT $\mathrm{LO}_{2}$ cable tray, the test encountered an instability (resonance) for $0.65<\mathrm{M}<0.80$. Study of this resonance has concluded that it results from three-dimensional unsteady ground plane interference effects. It is sensitive to Reynolds number and to model geometry (model gap/chord ratio) which explains the absence of the resonance in the original tests. While test results are valid outside this Mach number range, the goal of repeating the original stability analysis has not been possible since this Mach range encompasses the most critical conditions for the PAL ramps.

\section{Ground Vibration Testing of Cable Trays}

Ground Vibration Testing (GVT) of the flight standard design $\mathrm{LO}_{2}$ and $\mathrm{LH}_{2}$ cable trays was performed. Since there were no prior documented vibration tests of any cable trays, the primary objective was to identify the first two bending and torsion modes for each of the three selected sections of the trays. Primary focus was upon modal 
frequencies, mode shapes, and especially modal damping. Figure 13 shows the tray and shaker setup for the $\mathrm{LO}_{2}$ modal survey.

Features of the cable tray construction made repeatable vibration testing difficult. The trays are constructed as a three sided channel with a screwed-down cover lid. Also, to account for thermal effects only one end of each cable tray is securely bolted to the supports - the forward end for the $\mathrm{LO}_{2}$ trays and the aft end for the $\mathrm{LH}_{2}$ trays. Due to the inherent non-linear behavior of the structures, modal analysis was difficult. Pure plunging (bending) and pitching (torsion) motions were not found. Instead tray motions tended to involve coupled plunging motions, pitching motions, rocking on support brackets (roll motions), and sideways bending/swaying (lateral) motions.

Figure 14 shows the effect of shaker force level on the normalized Frequency Response Functions (FRF) of the $\mathrm{LO}_{2}$ tray. In Figure 14 the plunging mode of the $\mathrm{LO}_{2}$ tray is clearly seen at $\sim 162 \mathrm{~Hz}$ for the $1 \mathrm{lb}$ force but is heavily damped and shifted to higher frequency at the higher force levels. The pitching mode at $\sim 133 \mathrm{~Hz}$ is hard to detect for the $1 \mathrm{lb}$ force and is heavily damped at the higher force levels.

\section{OVERFLOW Full Stack Launch Configuration Steady CFD Computations and Selection of Wind Tunnel Test Conditions}

Three-dimensional full-stack launch configuration steady CFD analyses were used to define the flow conditions required to adequately test the $\mathrm{LO}_{2}$ and $\mathrm{LH}_{2}$ cable tray systems. The OVERFLOW Navier-Stokes computational aerodynamics method was used to perform these computations. The model used for this analysis is shown in Figure 15. The figure shows the surface grid used in the OVERFLOW calculations including the External Tank (ET), Solid Rocket Boosters (SRB), and the orbiter. The ET is shaded in gray, while the remaining components are shaded red. Two views of the surface grid are shown in the figure. The view on the left shows the right side of the vehicle, which for these analyses has been labeled as the "tray side," since it is the side of the ET that contains the $\mathrm{LO}_{2}$ and $\mathrm{LH}_{2}$ cable trays and the large Liquid Oxygen (LOX) Feedline. The right-hand figure shows the left side of the stack, which is referred to as the "clean side" since it is relatively free of external protuberances.

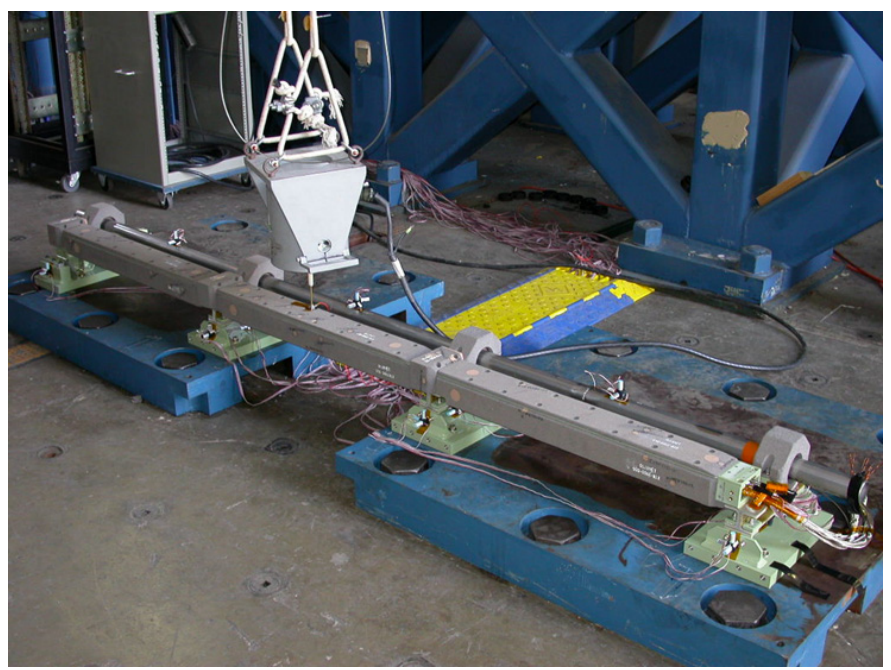

Figure 13. AEDC 16T $\mathrm{LO}_{2}$ Test Article and vibration test shaker installation.

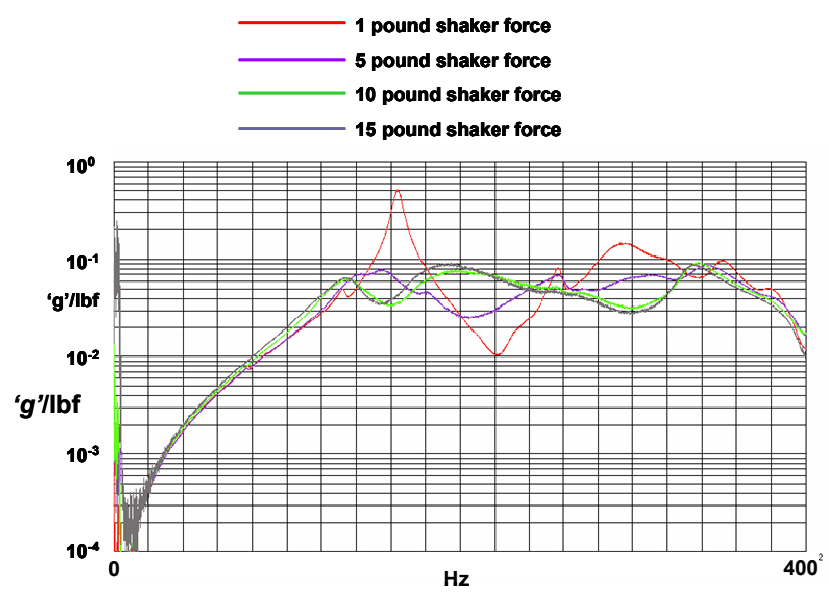

Figure 14. $\mathrm{LO}_{2}$ center cable tray section modal test drive point transfer functions (normalized) for the first "Flight" configuration as a function of force excitation level.
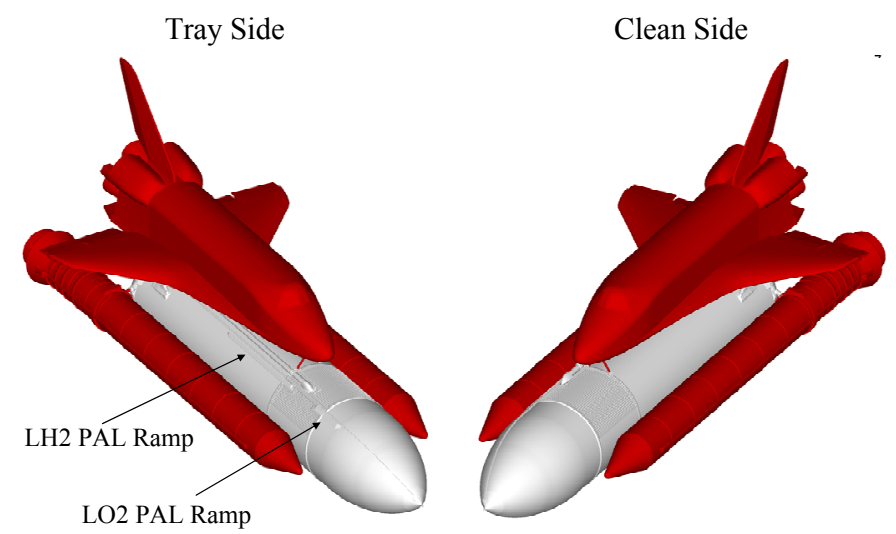

Figure 15. Surface grid for OVERFLOW model used to define AEDC test conditions.

10

American Institute of Aeronautics and Astronautics 
The primary objective of these analyses was to define local flow conditions in the vicinity of the cable trays that could be used to set conditions in the full-scale component testing performed at AEDC 16T. Since only a few sections of the cable trays and associated PAL ramps could be tested at AEDC, it was important to define the most extreme local flow conditions these components would encounter, including effects of the SRB, orbiter, and other ET protuberances, during launch. To define these extreme conditions a series of 54 full-stack OVERFLOW calculations were performed that adequately cover the portion of the launch trajectory where transonic flow over the cable trays is experienced.

Post processing of the OVERFLOW runs was a significant challenge since the grids are extremely large, and there were a large number of cases to be evaluated. A process by which the large amounts of data could be systematically reduced to a manageable level was developed. Flow data was extracted from the full-stack CFD solution in four "slabs" strategically positioned on the ET. Figure 16 shows the slab surrounding the $\mathrm{LO}_{2}$ cable tray system from which computed flowfield data was extracted for each run in the matrix. The $\mathrm{LO}_{2}$ slab extends from in front of the existing PAL ramp to behind the PAL ramp and includes the cable trays, $\mathrm{GO}_{2}$ repressurization line and the complete PAL ramp. The slab also extends vertically to a height approximately two times the cable tray height. A post-processing script was used to extract the CFD data enclosed in this slab and compute the maximum local Mach number and dynamic pressure as a function of the local crossflow angle. $\left(+90^{\circ}\right.$ being perpendicular to the tray from the Solid Rocket Booster to the stack centerline). The slab shown in Figure 16 is for the tray side of the tank. A similar slab of data weas extracted from the clean side of the tank, and was instrumental in the analysis of the flow conditions for the $\mathrm{LO}_{2}$ cable tray system. A similar CFD data processing operation was performed for the $\mathrm{LH}_{2}$ cable tray Test Article. This process was used to set wind tunnel freestream Mach number and dynamic pressure test requirements for the $\mathrm{LO}_{2}$ and $\mathrm{LH}_{2}$ cable tray Test Articles.

Figures 17 and 18 show the envelopes, including the additional margin required by the project for the $\mathrm{LO}_{2}$ and $\mathrm{LH}_{2}$ tray configurations, respectively. These figures were those used to set the AEDC 16T flow conditions for testing of the full-scale cable tray components. The $\mathrm{LO}_{2}$ conditions

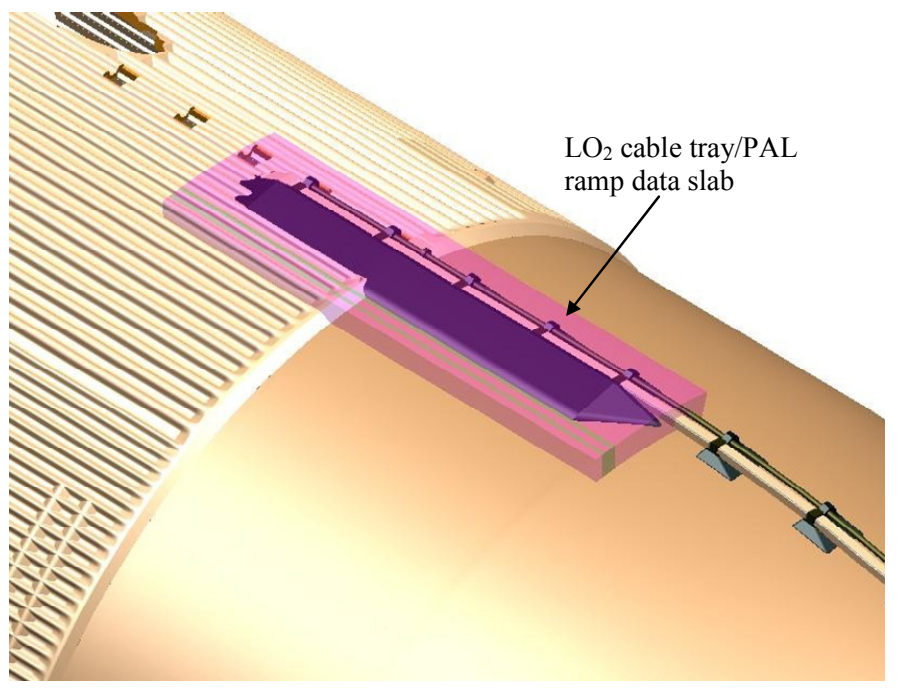

Figure 16. CFD data slab for the $\mathrm{LO}_{2}$ Cable Tray/PAL Ramp systems.

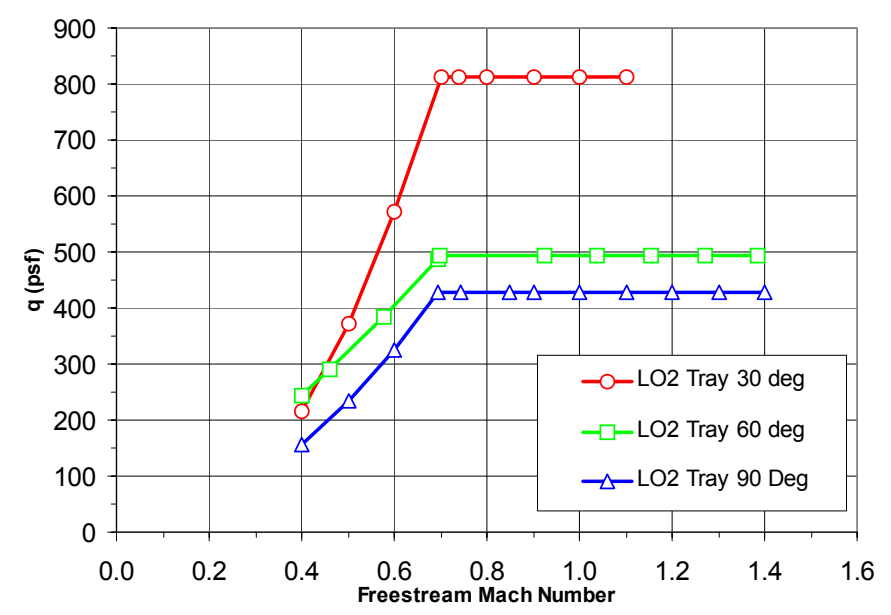

Figure 17. $\mathrm{LO}_{2}$ tray dynamic pressure freestream Mach number envelopes with additional structural and CFD uncertainty margin.

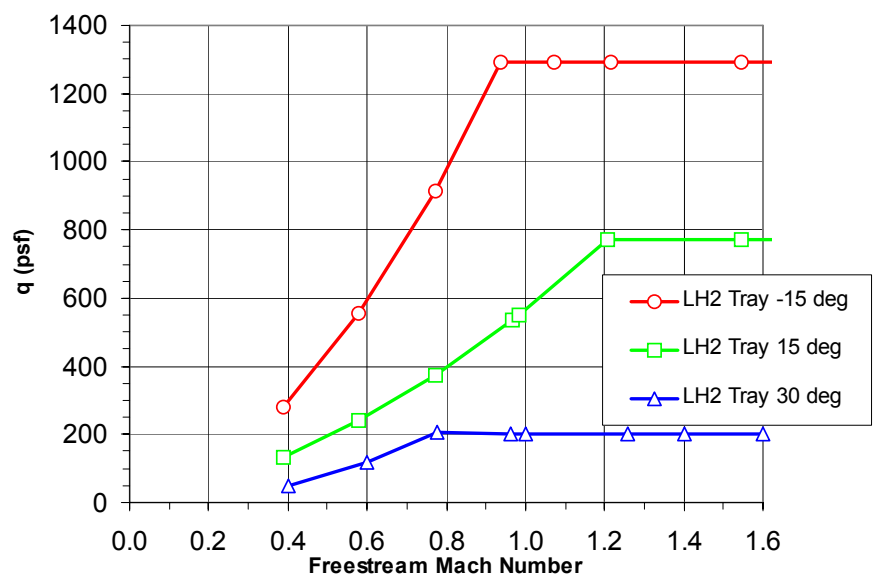

Figure 18. $\quad \mathrm{LH}_{2}$ tray dynamic pressure freestream Mach number envelopes with additional structural and CFD uncertainty margin.

11

American Institute of Aeronautics and Astronautics 
specified by these envelopes generally fall within the test envelope capabilities of the 16T wind tunnel. However, the maximum conditions for the three ramp configurations for the $\mathrm{LH}_{2}$ trays at -15 deg cross-flow fall outside the test envelope of the tunnel. For these configurations, the maximum dynamic pressure condition predicted by the CFD analysis could be attained, but the full margin conditions could not be met. These configurations were tested to a maximum dynamic pressure of $900 \mathrm{psf}$, which met the maximum condition predicted by CFD, but provides no margin

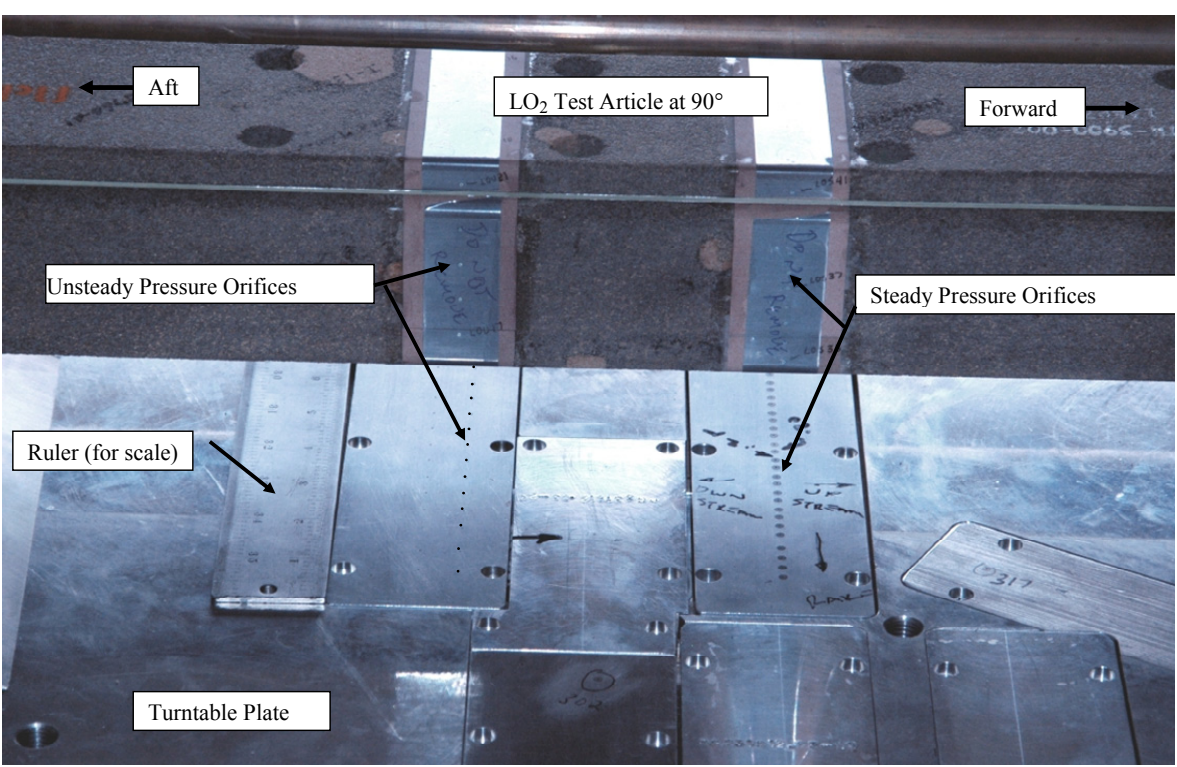

Figure 19. Steady and unsteady pressure orifices on $\mathrm{LO}_{2}$ Test Article center tray and on floor plate. beyond that condition.

Further dynamic analyses of cable tray responses to assumed unsteady loading conditions were performed to establish critical structural response levels. Using bandwidth limited Root-Mean-Squared (RMS) acceleration levels, along with known static loads, test termination criteria were established. A "Warning" RMS level was established based on a safety factor of 3 for each monitored accelerometer. A "Halt" RMS level was established based on a safety factor of 2 for each monitored accelerometer.

\section{AEDC 16T Test Results}

\section{A. Discussion of Aeroelastic Stability and Response Analysis}

Aeroelastic stability and aeroelastic response characterization rely upon a common basic analysis framework but differ in details. The classical bending-torsion flutter case results from interaction of two modes as the dynamic pressure increases. The modal frequencies approach each other ("coalesce"), the modal damping develops high sensitivity to dynamic pressure, and one of the modes becomes the unstable flutter mode while the other mode becomes more damped. Thus key features involved in such self-induced aeroelastic loading and which can lead to damaging instabilities include:

- Modal frequencies shifting with increasing dynamic pressure, especially frequencies which are coalescing;

- Modal damping decreasing (and increasing) with increasing dynamic pressure. Obviously, the lower damped modes draw the most attention.

In the case when there is external loading, the behavior of the structural response does not involve instability as in the case just discussed. Rather, the response is proportional to the amplitude of the external loading. Also, the modal frequency and damping may vary as dynamic pressure or Mach number are varied, but this behavior is independent of variation of the external loading. Several features which can distinguish this external forced response from a case involving an instability include:

- Little change in modal frequencies or damping for changing conditions;

- Little change in shape of spectral resonances (the damped, stable modes) even though their levels may show significant variation for changing conditions.

These observations provided the basis for monitoring the integrity of the cable trays during testing. 


\section{B. Test Overview}

The three test articles completed all of the planned configuration tests with no damage to their cable tray structures. Test Article 1, the current $\mathrm{LO}_{2}$ tray design, endured 40.8 hours of Air-On testing. Test Article 2, the $\mathrm{LO}_{2}$ tray with fence design, endured 6.9 hours of Air-On testing. Test Article 3, the $\mathrm{LH}_{2}$ tray design, endured 18.4 hours of AirOn testing. The actual time endured by cable trays during a launch in accelerating from Mach 0.6 to 1.6 is 35 seconds.

None of the nine cable tray sections (three tray section for each of the three Test Articles) experienced visible structural damage during the test. At the time this report was prepared, only the $\mathrm{LO}_{2}$ cable tray test article without fences had been dismantled and checked for internal structural anomalies, none of which were found. There was continued low level abrasion or erosion of the Super Lightweight Ablative (SLA) material covering the trays. Also, there were several instances of loss of larger amounts of SLA on the trays and on the SLA covering pressurization line "Barry mounts" of both $\mathrm{LO}_{2}$ cable tray test articles following significant amounts of test time.

\section{Steady Pressure Results}

Each cable tray test article was instrumented with static pressure ports in the center tray span. Figure 19 shows the location of the static pressure orifices on the $\mathrm{LO}_{2}$ cable tray without fence Test Article. Steady CFD computations for the full-stack shuttle launch configuration had shown that the boundary layer about the External Tank in the regions of the PAL
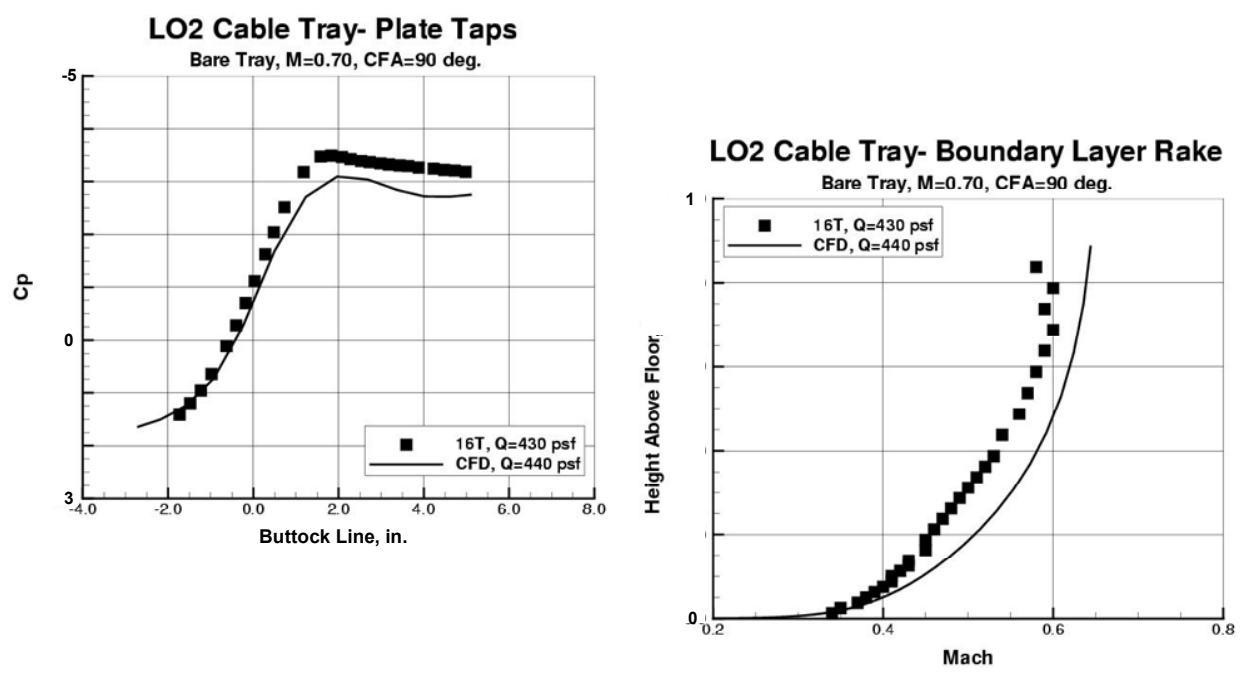

Figure 20. Comparison of experimental and CFD-calculated (normalized) floor plate static pressure and and boundary layer rake Mach number. $\mathrm{LO}_{2}$ cable tray Test Article, No Ramps, $M=0.70,90$ deg. crossflow angle.
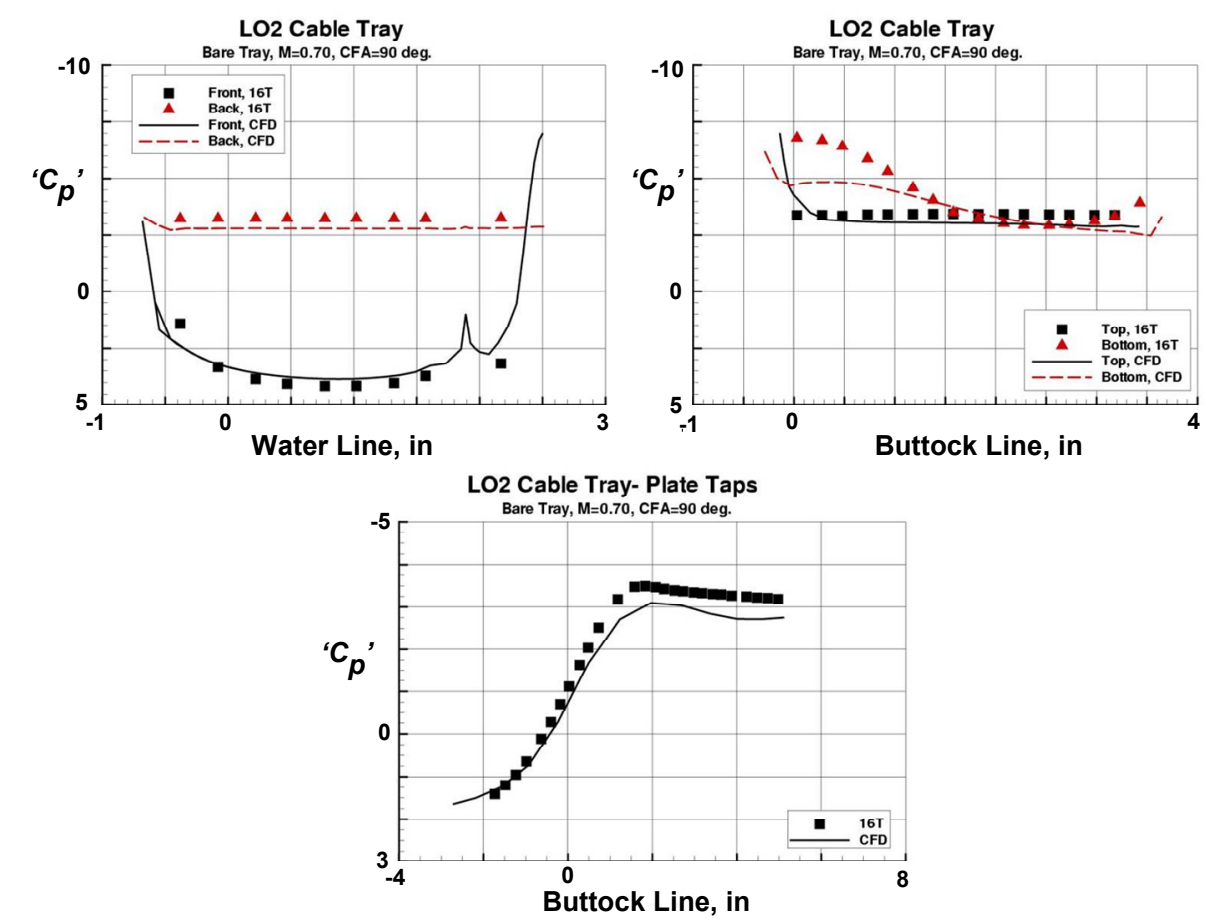

Figure 21. Comparison of experimental and CFD-calculated cable tray (normalized) static pressure. $\mathrm{LO}_{2}$ cable tray Test Article, No Ramps, $\mathrm{M}=\mathbf{0 . 7 0 , 9 0}$ deg. crossflow angle. 
ramps is approximately 6 inches thick, as was the floor boundary layer of the wind tunnel. Thus the cable trays were immersed in this turbulent, boundary layer flow. Figures 20 and 21 show good agreement of steady pressures computed with the OVERFLOW code for the $\mathrm{LO}_{2}$ cable tray wind tunnel test model geometry compared with static pressures from the test at $\mathrm{M}=0.70$.

\section{Unsteady Pressure Results}

Each cable tray test article was instrumented with unsteady pressure transducers in the center tray span. Figure 19 shows the location of the unsteady pressure orifices on the $\mathrm{LO}_{2}$ cable tray without fence Test Article. Tray unsteady pressures were integrated to compute sectional load on the middle tray of the Test Articles. Figure 22 shows the normalized sectional normal force across the Mach range of test conditions for the $\mathrm{LO}_{2}$ trays with No Ramps at 90 deg. crossflow and nominal pitch angle. Mean values are shown as filled symbols with standard deviation error bars. Minimum and maximum values of the sectional load are represented by triangle and gradient symbols, respectively.

\section{E. General Comments on Aeroelastic Response of Cable Trays}

For all test articles and configuration, RMS acceleration levels generally increased in going from PAL Ramps to Mini-Ramps and in going from MiniRamps to No Ramps. The response of the trays was different in a number of ways from the response of lifting surface aeroelastic test articles such as the wings and empennages of typical flutter models. These models are aerodynamically streamlined and designed such that flow separation is usually encountered only at the extremes of flight envelopes and well away from design conditions. The cable tray test articles on the other hand involve bluff body, separated flows at all conditions. Thus their response differs from that seen on typical flutter models which involve "selfinduced unsteady airloads" and exhibit "modal coupling" features which can lead to instabilities. Rather, the behavior of the trays is best understood as response to external, forced excitation.

There is not a comprehensive understanding of the origins of these forcing excitations. There is relatively little unsteady or aeroelastic experimental data for bodies with geometries similar to these test articles and for these transonic test conditions. The focus of the test was upon the center trays of the test articles with the forward and aft trays presumed to provide adequate in-flow boundary conditions for the center tray. Also, there was a focus upon the mid-span region of the center trays. It was assumed that this would be the region of largest response and best representative of two-dimensional crossflow properties. Hence it is here that the large majority of instrumentation was located. Only two accelerometers were

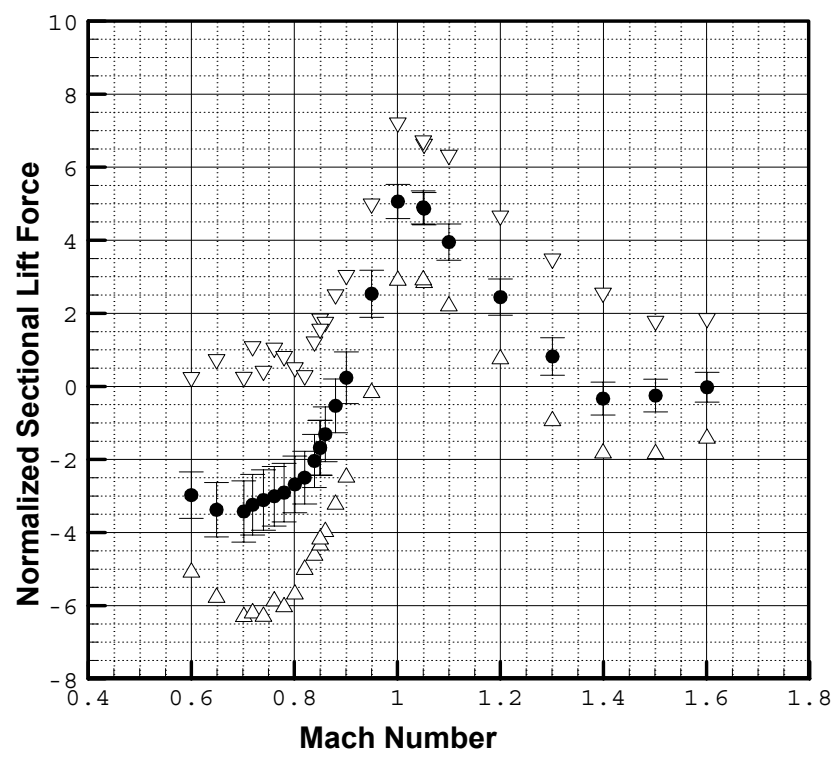

Figure 22. $\quad \mathrm{LO}_{2}$ tray normalized sectional normal force; $\mathrm{LO}_{2}$ Trays at $90^{\circ}$ crossflow, No Ramps, nominal pitch angle.
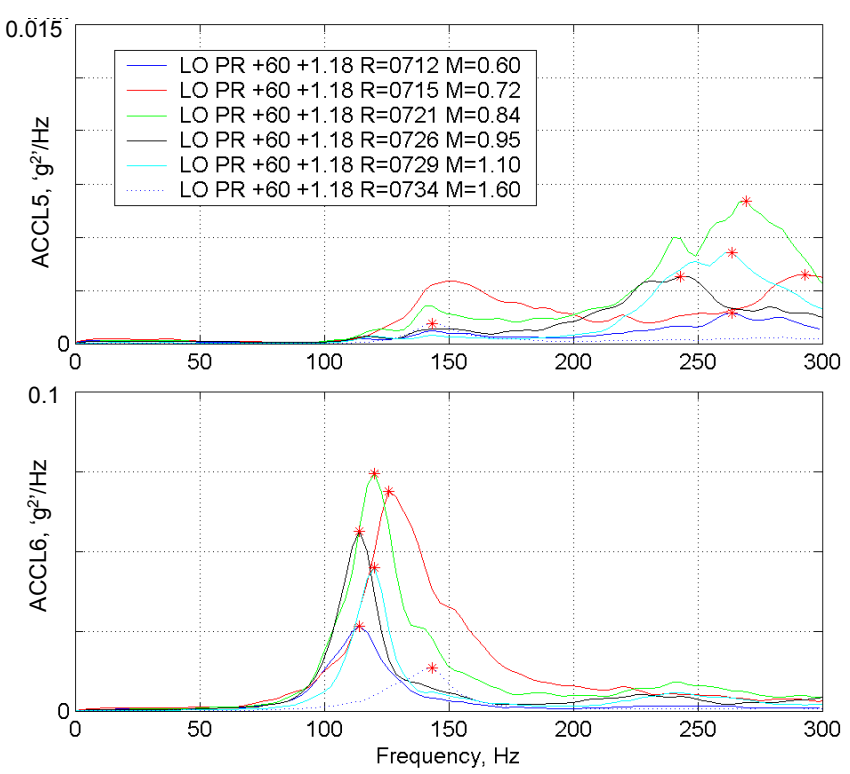

Figure 23. Normalized power spectral density functions for ACCL5 and ACCL6 for several Mach numbers, from AOP 10. $\mathrm{LO}_{2}$ cable trays with PAL Ramps at $60 \mathrm{deg}$. crossflow and nominal pitch angle. 
located in each of the outer trays (at mid-span) and there was no pressure instrumentation away from the mid-span of the center trays.

It is thus interesting that the test revealed that a good deal of activity occurs in other regions of the test articles. Here attention can be drawn to the regions of the center tray adjacent to the forward and aft ice/frost ramps.

The origins of the forced excitation felt by the trays can be broadly described as:

- Unsteadiness or turbulence in the oncoming flow/crossflow. The entire height of the cable trays is within the boundary layer of the External Tank;

- Unsteadiness associated with flow separations about the trays and ice/frost ramps;

- Leading-edge bubbles forming at the leading edge of the tray top and bottom;

- Unsteadiness of the separated wake region about the tray/pressurization line(s) - Strouhal wake vortex shedding; - Unsteadiness in gap flow, possibly involving unsteady shock/boundary layer

interactions.

A surprising feature of the tray responses is the appearance of quite localized response regions (within the center trays, where this could be observed due to the number of accelerometers). There are many instances of response at modal frequencies seen on only one or two adjacent accelerometers with little or no response at neighboring sensors.

\section{F. Cable Tray Modal Behavior Studied with Power Spectral Density Functions}

The modal behavior of the cable trays was studied using Power Spectral Density (PSD) analysis of the accelerometer signals. This allows identification of the modal content of the tray response by comparison with the GVT test and FEM analysis results. Primary interest was focused upon the lowest frequency modes. The nature of the cable tray construction does not result in simple plunging and pitching modal motions. Rather, the motions observed involved highly coupled plunging, pitching, and thrusting motions. The magnitude, frequency, and damping of modal peaks were studied for varying test conditions to note trends or constancy. Thus a pitch mode spectral peak increasing in amplitude and narrowing in width may be indicative of an approaching instability whereas a spectral peak feature increasing in amplitude but with little change in peak width (damping) or frequency is indicative of increasing external airloads with no issue regarding stability.

Figures 23-25 are representative of typical PSD content observed in the test results. PSD's of ACCL5 and ACCL6
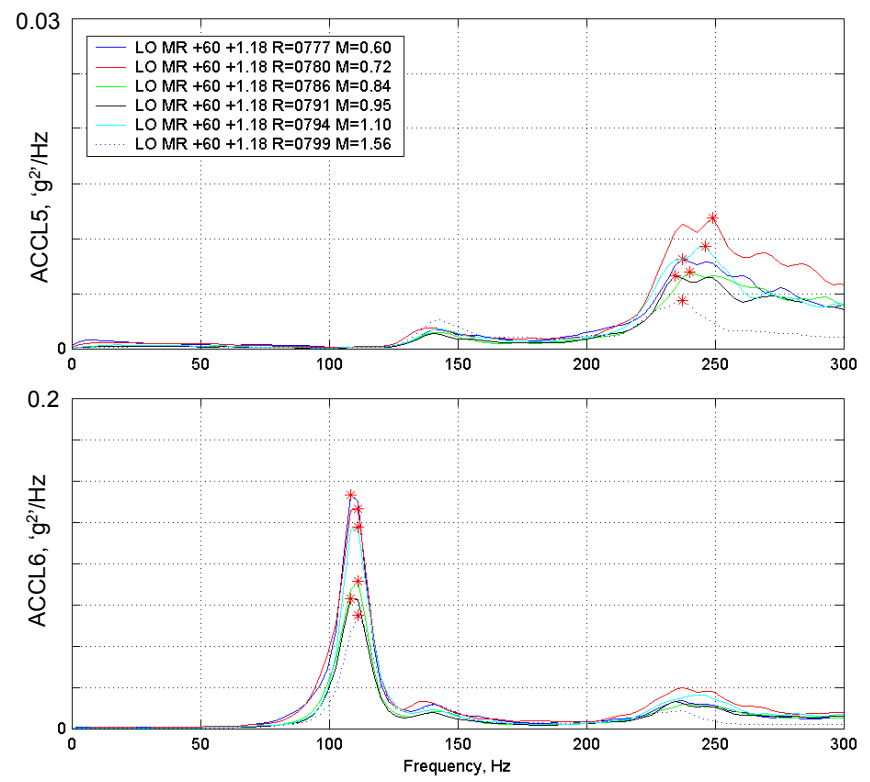

Figure 24. Normalized power spectral density functions for ACCL5 and ACCL6 for several Mach numbers, from AOP $11 \mathrm{LO}_{2}$ cable trays with Mini Ramps at $60 \mathrm{deg}$. crossflow and nominal pitch angle.
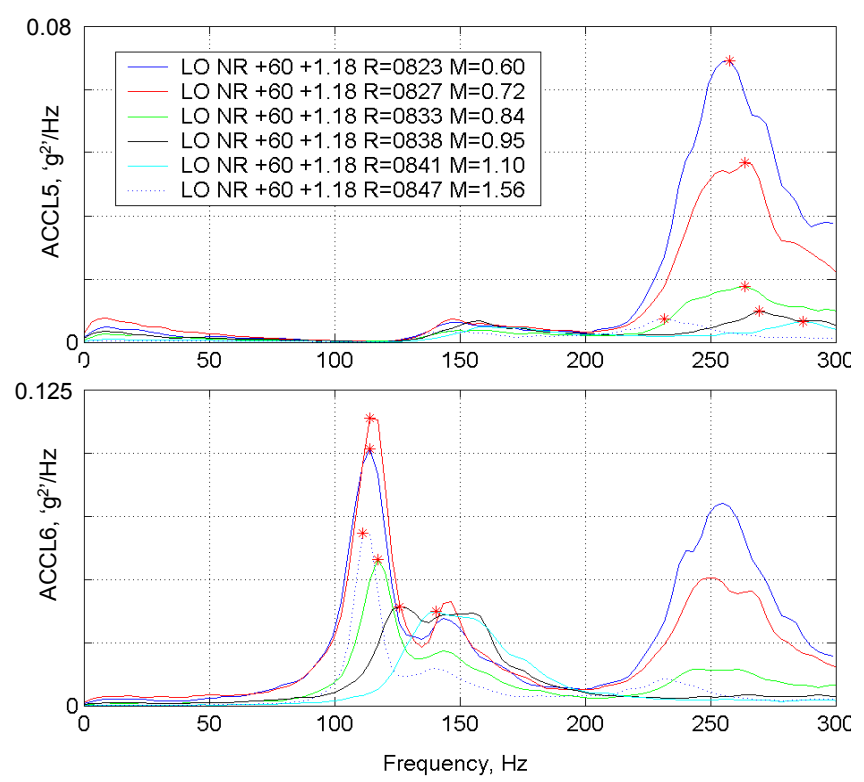

Figure 25. Normalized power spectral density functions for ACCL5 and ACCL6 for several Mach numbers, from AOP 13. $\mathrm{LO}_{2}$ cable trays with No Ramps at $60 \mathrm{deg}$. crossflow and nominal pitch angle.

15

American Institute of Aeronautics and Astronautics 


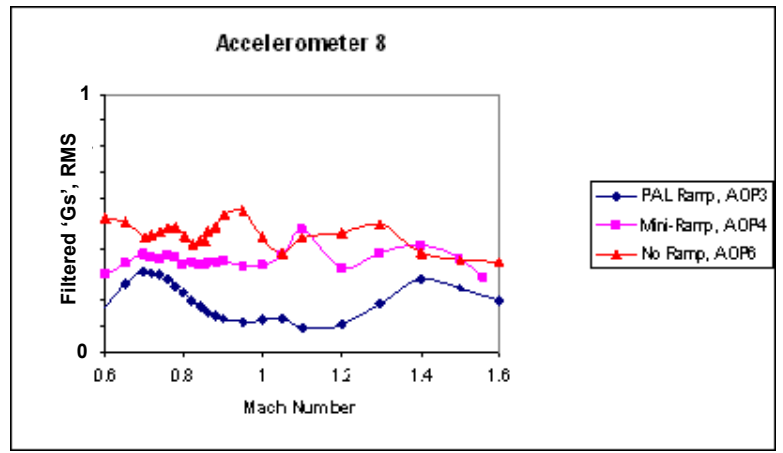

Figure 26. Comparison of normalized RMS levels from selected accelerometers versus Mach number and ramp configuration for $\mathrm{LO}_{2}$ cable trays (Test Article 1). 90 deg. crossflow and maximum dynamic pressures.

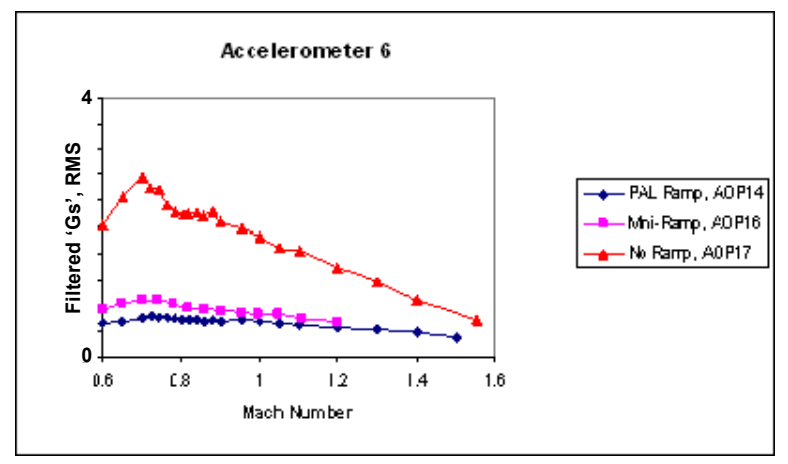

Figure 28. Comparison of normalized RMS levels from selected accelerometers versus Mach number and ramp configuration for $\mathrm{LO}_{2}$ cable trays (Test Article 1). 30 deg. crossflow and maximum dynamic pressures.

are given for the $\mathrm{LO}_{2}$ Cable Tray without fence Test Article at 60 degres No Ramps. Frequency Response Functions are given for $0.6<\mathrm{M}<1.60$. The primary response is seen in accelerometer ACCL6 in the range of 120-140 Hz. This is the 'pitch/roll' mode measured during the GVT testing, in which the tray motion is coupled pitching and rolling with the outboard tray region showing little normal motion. There are modest shifts in the modal frequency but little change in the apparent modal damping as noted in the width of the modal resonances. Also, noticeable changes in the peak levels of modal features were observed for different configurations of this Test Article (e.g. changing from PAL Ramps to No Ramps, or changing crossflow angle). However, there was no indication of significant loss of damping or modal coupling. Small-to-noticeable shifts in modal frequencies were seen. Similar frequency shifts had been observed during the wind-off GVT testing and are to be expected for such heavily damped structures with nonlinear elements such as joints with freeplay.

Similar modal behavior was seen in the responses of the other two Test Articles. Modest variations in modal frequencies and damping occur for changes in crossflow angle, Mach number, and ramp configuration. Some larger variations in modal response levels are noted which are attributed to variations in external airload force levels. Modal damping levels are moderate to large and no trends were observed of significantly decreasing damping.

\section{G. Root-Mean-Square Accelerometer Response Levels}

In this section, representative samples of normalized accelerometer response RMS levels versus Mach number will be given. Generally levels from ACCL6 are shown. Figures 26-28 compare results from the $\mathrm{LO}_{2} \mathrm{Cable}^{\mathrm{T}} \mathrm{Tays}$ for the three ramp configurations, all for the maximum dynamic pressures tested. Generally, RMS response levels increased modestly going from PAL Ramps to Mini Ramps to No Ramps. The largest responses were seen for the No Ramps configuration at 30 degrees Figure 28) where some interaction with Strouhal vortex shedding may be 
involved.

A second method of analysis of the RMS response levels involved comparison of results from varying dynamic pressure for each configuration, with the lower dynamic pressure results scaled to the maximum dynamic pressures tested. A linear variation of response levels with dynamic pressure is an indication of forced response, termed buffeting, to external excitation and that self-induced unsteady airloads are not involved. Aeroelastic instabilities such as flutter are due to self-induced unsteady airloads. Figure 29 presents a sample of this approach. The case corresponds to the No Ramp results at 30 degrees crossflow shown in Figure 28. The near coincidence of the scaled and unscaled response levels is indicative of linear behavior and counter-indicative of any instability development. This linear relationship was seen in most of the measurements. Where small or modest differences were seen, the scaled (lower) dynamic pressure levels were generally larger which emphasizes the favorable action of nonlinear damping (i.e. higher damping for larger motions).

Details presented in Figures 23 through 29 are necessarily a limited sample of the measured structural responses of the cable tray Test Articles. However, similar observations and comments regarding the modal behavior seen in PSD analysis and the tray acceleration levels seen in RMS analysis can be made for all of the tested configurations.

\section{Summary}

Three Test Articles consisting of flight standard design Space Shuttle External Tank cable tray sections and several Protuberance Aerodynamic Load (PAL) Ramp shielding devices were tested in the USAF AEDC 16T transonic wind tunnel. The effects of many components of the Shuttle launch configuration (External Tank and its flexibility, Solid Rocket Boosters, Shuttle Orbiter, etc.) were not included. Thus the test was an aeroelastic stability and response characterization test of the cable trays rather than an aeroelastic clearance test.

No aeroelastic instabilities or damaging response levels were encountered for any of the configurations tested. The two $\mathrm{LO}_{2}$ cable tray Test Articles achieved the desired test dynamic pressures and Mach numbers, including 45 percent margin, for all ramp configurations. Thus any of the alternative $\mathrm{LO}_{2}$ cable tray ramp configurations - No Ramps, Mini Ramps, or trailing-edge fences - are viable alternatives to the existing PAL Ramps.

Experience for the $\mathrm{LH}_{2}$ cable tray Test Article was similar although wind tunnel test envelope limitations prevented testing to the desired margin of dynamic pressure. Still, the configurations were tested to the maximum predicted dynamic pressures experienced during launch. Again, no aeroelastic instabilities or damaging response levels were encountered.

The remaining steps in determining if there are adequate margins to implement changes to the current PAL Ramps are: i.) Acquisition of Flight (launch) Data, and ii.) Structural Assessment of the proposed modifications. Dynamic response flight data from Shuttle launches must be obtained and studied to establish the behavior and understanding of the current cable trays with the existing PAL ramps. Measured response details, such as modal characteristics and amplitude levels, must be consistent with those obtained during the AEDC 16T wind tunnel test in order to validate the Structural Assessments. Flight design criteria, factors of safety, allowable tolerances, and required margins relevant to flight design standards must be addressed in the Structural Assessment.

\section{Acknowledgments}

The Space Shuttle External Tank PAL Ramp/Cable Tray Evaluation project has enjoyed excellent support and participation from many organizations and individuals. The authors wish to acknowledge the contributions of Terry F. Greenwood, Darren K. Reed, C. Mark Seaford, Kathy O. Kappus, and Alan Droege of NASA Marshall Space Flight Center; Richard C. Smith, Edgar A. Rawls, Camille McConnell, Paul Jordan, Daniel Fontenot, and J. Peter Reding (ret.) of Lockheed Martin Space Systems Company; William Crosby of Sverdrup Corp.; and Reggie Riddle, James Solomon (ret.), Rod Stewart, and Jacob Cashion of the Aerospace Testing Alliance at the USAF AEDC.

\section{References}

${ }^{1}$ Ericsson, L. E. and Reding, J. P., “Aeroelastic Analysis of the Space Shuttle External Tank Cable Trays,” Final Technical Report, Contract ASO-751485, LMSC D766543, April 1981, Lockheed Missiles \& Space Company, Inc., Sunnyvale, California.

${ }^{2}$ Ericsson, L. E. and Reding, J. P., "Aeroelastic Characteristics of the Space Shuttle External Tank Cable Trays,” AIAA Paper No. 82-0633, $23^{\text {rd }}$ Structures, Structural Dynamics and Materials Conference, New Orleans, LA, May 10-12, 1982.

${ }^{3}$ Ericsson, L. E. and Reding, J. P., "Aeroelastic Analysis of the Light Weight $\mathrm{LH}_{2}$ Cable Tray," LMSC D059511, October 1983, Lockheed Missiles \& Space Company, Inc., Sunnyvale, California. 